\title{
NeuroImage
}

\section{Levels of appraisal: A medial prefrontal role in high-level appraisal of emotional material}

\author{
Raffael Kalisch, ${ }^{*}$ Katja Wiech, Hugo D. Critchley, and Raymond J. Dolan \\ Wellcome Department of Imaging Neuroscience, Functional Imaging Laboratory, Institute of Neurology, University College London, \\ London WCIN $3 B G$, UK
}

Received 4 July 2005; revised 21 October 2005; accepted 14 November 2005

Available online 4 January 2006

\begin{abstract}
Appraisal refers to the evaluation of the meaning of emotional stimuli and is considered causal in the generation of an emotional response. Cognitive neuroscience has paid little attention to a theoretical distinction between low-level appraisal, considered to be automatic and preattentive, and high-level appraisal that requires attentional and working memory resources. To disentangle low-level from high-level appraisal, we varied cognitive load in a concurrent, unrelated working memory task, while anxiety was induced through anticipation of impending pain. Confirming theoretical predictions, we show that anxiety-related activity in dorsal medial prefrontal/rostral anterior cingulate cortex (dorsal MPFC/ACC) is attenuated under high, relative to low, cognitive load. Lateral prefrontal regions previously implicated in reappraisal and cognitive emotion regulation show a similar interaction between anxiety and cognitive load. Critically, there were no changes in physiological and subjective measures of low-level appraisal outcome and emotional response generation as a function of load, allowing us to conclude that MPFC/ACC and lateral PFC activity during anticipatory anxiety reflects high-level appraisal. Our data provide neurobiological evidence for a distinction between low-level and high-level appraisal mechanisms.
\end{abstract}

(C) 2005 Elsevier Inc. All rights reserved.

\section{Introduction}

The evaluation of emotional material is thought to involve both low-level and high-level appraisals (Frijda, 1993; Lazarus, 1966; Leventhal and Scherer, 1987; Öhman, 1993; Reisenzein, 2001; Robinson, 1998; Smith and Kirby, 2001). Low-level appraisal is often described as non-conscious, preattentive, automatic, effortless, nonpropositional and "hard-wired", referring to a strong hereditary component (e.g., Leventhal and Scherer, 1987; Robinson, 1998). An example is the computation of probabilistic predictions of reward or punishment during conditioning and the later retrieval of conditioned memories (Dayan and Abbott, 2001).

\footnotetext{
* Corresponding author. Fax: +44 2078131420.

E-mail address: rkalisch@fil.ion.ucl.ac.uk (R. Kalisch).

Available online on ScienceDirect (www.sciencedirect.com).
}

High-level appraisal is described as conscious, controlled and requiring attentional and working memory resources (Leventhal and Scherer, 1987; Robinson, 1998). As such, it is compromised under high cognitive load (Schneider et al., 1984). An example is reward or punishment expectation based on contingency awareness formed during conditioning.

While low-level appraisal may allow for primitive, "quick and dirty" responses to evolutionarily primed stimuli (LeDoux, 1996), high-level appraisal is more elaborate and lends flexibility to behavior (Scherer, 1984). A potential link between both levels of appraisal is the often attention-grabbing or "intrusive" nature of low-level appraisal outcomes (Christianson, 1992; Erdelyi and Appelbaum, 1973; Moray, 1959; Nielsen and Sarason, 1981). Low-level appraisal may generate subjective emotional experience and attentional orientation toward emotional stimuli with a purpose to inform our conscious awareness of a relevant situation as well as to recruit high-level appraisal processes (e.g., Clore and Parrott, 1991).

Whereas the investigation of the neural basis of different appraisal dimensions such as novelty or valence (reviewed in Sander et al., 2005) has received much interest from cognitive neuroscientists, less attention has been paid to the neurobiological study of levels of appraisal. Researchers interested in low-level appraisal have made considerable effort to show that certain appraisals are independent of consciousness (e.g., LeDoux, 1996; Öhman, 2005). However, a neurobiological distinction between low-level and high-level appraisal mechanisms has not been established.

While controlled processing is generally associated with prefrontal cortex, the medial prefrontal cortex in particular may be a key structure in high-level appraisal. The MPFC/ACC is involved in attention to emotional stimuli (Fichtenholtz et al., 2004; Vuilleumier et al., 2002); emotional awareness (Lane et al., 1997, 1998; Simpson et al., 2001); rating of affect intensity (Taylor et al., 2003); and explicit judgments of self relevance (Blackwood et al., 2004; Fossati et al., 2003; Johnson et al., 2002; Kelley et al., 2002; Phan et al., 2004). The MPFC/ACC may thus be at the interface between low- and high-level appraisal, providing access to consciousness for low-level appraisal outcomes or, alternatively, 
may mediate high-level appraisal (Lewis, 2005; Sander et al., 2005).

A general problem in previous studies is that a correlation between high-level appraisal processes and MPFC/ACC activity does not imply a direct causal link. Instead, correlations may be caused by a third factor linked to both. For example, MPFC/ACC activity during high-level appraisal may be a result of parallel lowlevel appraisals potentially involving the MPFC/ACC (Seymour et al., 2004). MPFC/ACC-based functions such as the generation of autonomic or motor responses (Critchley, 2004; Devinsky et al., 1995) may also be activated alongside high-level appraisal processes. These potential confounds need to be ruled out when trying to demonstrate that a brain area subserves high-level appraisal.

One of the best replicated findings in emotion research is that low-level appraisal directly impacts on autonomic activity, behavior and subjective feelings, without the need for high-level cognitive appraisal as a mediator (Kunst-Wilson and Zajonc, 1980; Lazarus and McCleary, 1951; LeDoux, 1996; Öhman, 2005; Robinson, 1998; Robles et al., 1987). Hence, autonomic indices and self report can be used to measure changes in low-level appraisal outcome. Inference about motor response generation is generally complicated by experimentally imposed needs to inhibit overt behavior such as approach or withdrawal responses. However, where response inhibition requirements are identical under different experimental conditions, indices of action-supporting autonomic (sympathetic) activity can serve as an indirect measure for covert action tendencies. Thus, if one could dissociate changes in high-level appraisal activity from changes in autonomic and self report indices, this would allow for a more specific ascription of brain activation.

High-level appraisal is an invariable response to consciously perceived emotional stimuli (Epstein and Roupenian, 1970; Gross and John, 2003; Hare, 1966; Houston and Holmes, 1974; Monat et al., 1972). To selectively interfere with spontaneous high-level appraisal processes, we manipulated attention and working memory load (cognitive load) via the difficulty of a concurrent unrelated task (n-back task), while anxiety was induced by anticipation of impending pain (see Fig. 1 for design). It was assumed that high-level appraisal of anxiogenic stimuli (pain cues), and of the elicited bodily and subjective anxiety reactions, would be reduced in a High Load relative to a Low Load condition. Importantly, prior studies had indicated that such a cognitive manipulation has little if any effect on autonomic and subjective experiential measures (Chua et al., 1999; Houston and Holmes, 1974). We thus expected to find the dissociation defined above as a criterion for high-level appraisal specificity.

At a neural level, involvement of the MPFC/ACC in high-level appraisal should be evident from a reduction of anxiety-related $\mathrm{MPFC} / \mathrm{ACC}$ activity during high relative to low cognitive load. In the analysis, we focused on a dorsal MPFC/ACC region, within Brodmann areas 24 and 32, which is reproducibly found in studies of anticipatory anxiety (see Materials and methods). a

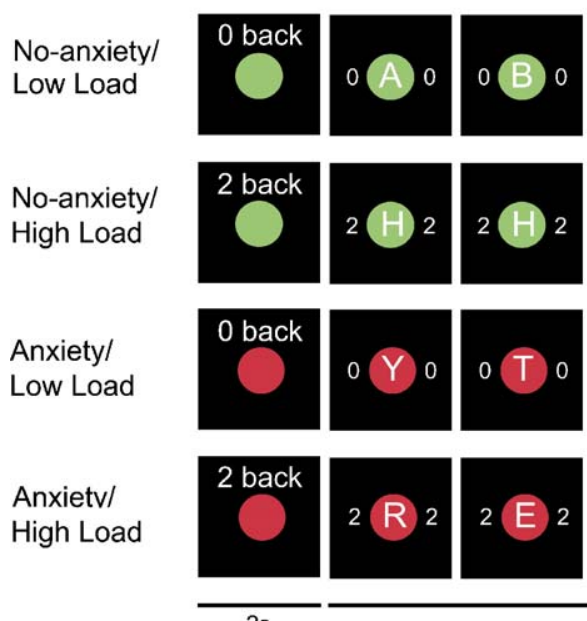

2s

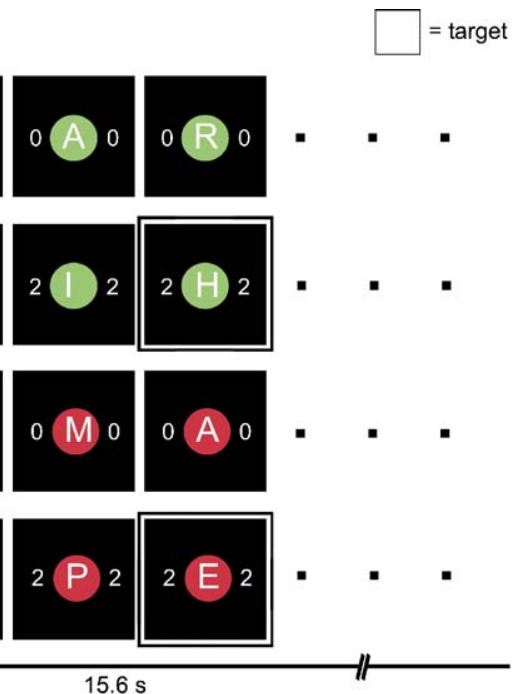

b

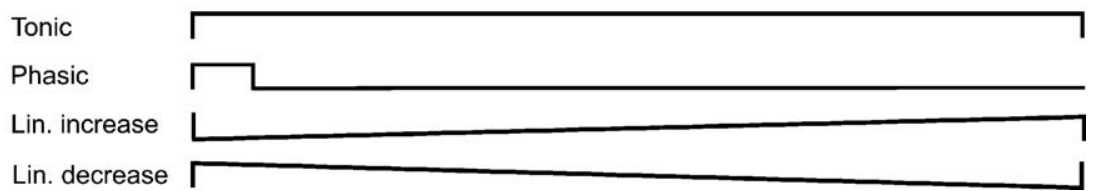

Fig. 1. Design and analysis. (a) In an adapted version of a previously employed anticipatory anxiety paradigm (Kalisch et al., 2005), subjects were forewarned that they might receive an electric pain stimulus to the hand at a probability of $25 \%$ at any time during a 15.6 -s epoch (Anxiety condition, red dots). During a control condition (No-anxiety, green dots), subjects knew that they would not be stimulated. Cognitive load was manipulated by a 2-back task (High Load condition). In the corresponding control condition (Low Load), subjects performed a modified version of the 0-back task. The study involved a $2 \times 2$ factorial design, with factors Anxiety (No-anxiety vs. Anxiety) and Load (Low Load vs. High Load). There were thus four experimental conditions: No-anxiety/Low Load; No-anxiety/High Load; Anxiety/Low Load; and Anxiety/High Load. In this design, a reduction of anxiety-related activity should be evident from the interaction term $(\text { Anxiety }>\text { No-anxiety) })_{\text {Low Load }}>$ (Anxiety $>$ No-anxiety) $)_{\text {High Load. }}$ (b) Neural activations during blocks of Anxiety/No-Anxiety were modeled as tonic, phasic and linearly increasing and decreasing responses (see Materials and methods). (For interpretation of the references to colour in this figure legend, the reader is referred to the web version of this article.) 


\section{Materials and methods}

\section{Participants}

Fourteen right-handed healthy normal subjects (mean age 30 years, age range 18-58 years, 9 female) participated in the experiment. The subjects were pre-assessed to exclude those with a prior history of neurological or psychiatric illness, including anxiety disorders. Subjects' scores for trait and state anxiety (obtained prior to scanning using questionnaires STAI-S and STAIT, Mind Garden, Redwood, CA, USA) were $35.3 \pm 2.5$ (SEM) and $39.4 \pm 1.9$, respectively, and thus deviated less than one standard deviation from a normal working adult population (Spielberger, 1983). Scores on a cognitive failures questionnaire (CFQ; Broadbent et al., 1982) were $38.3 \pm 3.0$ and thus also corresponded to general observations. All subjects gave informed consent, and the study was approved by the Joint Ethics Committee of the National Hospital for Neurology and Neurosurgery.

\section{Experimental design}

Anxiety was induced by anticipation of a painful electric stimulus. During an instruction epoch of $2 \mathrm{~s}$, subjects were shown a red dot in the center of the screen (Fig. 1). The instruction epoch also served to inform subjects about the nature of the upcoming task (0-back or 2-back, see below). The instruction was followed by a 15.6-s epoch during which subjects had to perform the 0 - or 2back task while anticipating a potential pain stimulus. During the whole $15.6 \mathrm{~s}$, the red dot remained in the center of the screen to constantly remind subjects of the threat. Subjects were told that the probability of receiving a painful stimulus was $25 \%$ for each of these 15.6-s epochs and that the stimulus could occur at any time during the $15.6 \mathrm{~s}$. A control condition (No-anxiety) during which subjects knew they would not be stimulated was equivalently signaled by a green dot. Pain stimuli were applied to the back of the right hand using a custom-built electrical stimulator delivering 20 or $100 \mathrm{~Hz}$ trains of electrical pulses (4 ms monopolar square waveform pulses, $1 \mathrm{~s}$ duration, 0.1 to $6 \mathrm{~mA}$ ) through a silver chloride electrode.

Cognitive load was operationalized by a 2-back task (High Load condition) vs. a 0-back task (Low Load condition). During the 2-s instruction epoch, subjects either saw "2-back" or "0-back" above the colored (red, green) dot signaling Anxiety or No-anxiety (Fig. 1). During the following 15.6-s epoch, a sequence of eight white capital letters, each presented for $1 \mathrm{~s}$ with an inter-stimulus interval of $1 \mathrm{~s}$, was shown on the background of the colored dot. In the 2-back task, subjects pressed a button with their right middle finger to indicate when a letter was identical to the letter shown " 2 back"; for any other letter, they made a button press with their right index finger. In the 0 -back task, subjects made a button press with their right index finger for any letter. Trials were separated by intertrial intervals (ITI) of 6 to $7.4 \mathrm{~s}$ during which a fixation cross was shown.

The experiment was divided into 3 runs of 24 trials, with duration of approximately $10 \mathrm{~min}$ per run. The number of trials per experimental condition (excluding those where subjects actually received pain) inter-individually varied between 12 and 15 (mean 14.3; No-anxiety/Low Load, No-anxiety/High Load) and 11 and 19 (mean 15.9; Anxiety/Low Load; Anxiety/High Load), respectively. The sequence of trials was randomized. After 24 out of the 72 trials
(6 per condition, randomized), subjects rated the anxiety experienced during the preceding trial on a 100-point visual analog scale using left and right buttons to move a pointer on a horizontal bar ("How anxious did you feel during this trial?"). They were explicitly told before the experiment not to rate their affective response to the actual pain.

\section{Imaging}

A 3 T MR head scanner (Magnetom Allegra, Siemens, Erlangen, Germany) was used to acquire gradient echo T2*weighted echo-planar images (EPI) images with BOLD (blood oxygenation level-dependent) contrast $(\mathrm{TE}=30 \mathrm{~ms}, \mathrm{TR}=2.86 \mathrm{~s}$, flip angle $=90^{\circ}$, slice tilt $=30^{\circ}, \mathrm{z}$-shim gradient prepulse $=-1 \mathrm{mT} /$ $\mathrm{m} \mathrm{ms}$ ). Each volume comprised of 44 oblique axial slices of $2 \mathrm{~mm}$ thickness and $3 \times 3 \mathrm{~mm}^{2}$ in-plane resolution with a slice distance of $1 \mathrm{~mm}$. A total of 223 volumes per run were acquired. These parameters produced EPI images in which signal dropout due to susceptibility-induced field inhomogeneities was minimized for orbitofrontal cortex (Deichmann et al., 2003). Subjects were placed in a light head restraint within the scanner to limit head movement during acquisition. A T1-weighted structural image was also acquired (3D MDEFT; Deichmann et al., 2004).

\section{Procedure}

Upon arrival, subjects received written task instructions and completed STAI and CFQ questionnaires. Subjects were then given up to $10 \mathrm{~min}$ of practice without pain stimulation on a PC outside the scanner. This was followed by a pain calibration procedure which assured that current levels and stimulation frequencies were chosen which induced intermediate subjective anxiety. To achieve this, subjects verbally rated their anxiety experienced during a 16-to-0 countdown during which they knew they might receive a previously experienced painful stimulus at any time at $25 \%$ probability. The countdowns were repeated with different current levels, starting at low levels, until an anxiety level between 30 and 60 (on a 100-point scale) was reached. Current levels were adjusted between experimental runs if subjective ratings in the Anxiety minus No-anxiety comparison during the Low Load condition differed markedly from the previously calibrated value. Inside the scanner, another short practice run $(8$ trials) without pain stimulation was performed to familiarize subjects with the scanning procedure followed by the three experimental runs. Heart rate (HR) was monitored using a pulse oximeter (Nonin 8600FO, Nonin Medical, Plymouth, MN, USA); the pulse probe was placed on the index finger of the left hand. After the experiment, subjects were asked how stressed they were and how much effort they made to perform the task (from 1 to 5) during each of the four experimental conditions. One subject did not provide stress ratings.

\section{Data analysis}

Raw HR waveforms were visually inspected and HR data were excluded where automatic pulse detection was inaccurate (1 subject). Average HR level (HRL) of the 2-s instruction epoch plus the 15.6-s task epoch were normalized to ITI; initial phasic heart rate responses (HRR) were taken from 1 to $6 \mathrm{~s}$ after start of instruction and normalized identically. Blocks where subjects actually received pain stimuli were excluded from the analysis. 
Heart rate responses (HRR) to pain stimulation were calculated as average heart rate during the post-stimulus time window (of 1-s duration) which showed maximum physiological activation in the group data (from 2000 to $3000 \mathrm{~ms}$ ), normalized to the second prior to stimulus. Statistical inference was based on paired $t$ tests and one-way analysis of variance (ANOVA) with repeated measures within SPSS 11.

Imaging data were analyzed using SPM2 (www.fil.ion.ucl. ac.uk/spm/spm2; Ashburner et al., 2004). The 5 initial images of each run were discarded to account for T1 equilibration. To account for motion artefacts, images were realigned to the sixth volume of the first run. Images were spatially normalized to a standard $\mathrm{T} 2 *$ template, spatially smoothed using a Gaussian kernel with a full width at half maximum (FWHM) of $6 \mathrm{~mm}$, temporally high-pass-filtered (cut-off $128 \mathrm{~s}$ ) and corrected for temporal autocorrelations using first-order autoregressive modeling. Statistical analysis was carried out by applying a random effects analysis using the general linear model across the 14 subjects. Each of the four experimental conditions was modeled using three different temporal profiles of neuronal response during the 17.6-s epoch of combined instruction and task: a phasic response occurring at the beginning of the epoch, a tonic neuronal response lasting the whole duration of the epoch and a linearly increasing response across the epoch (Fig. 1b). Multiplication of the linearly increasing regressor by -1 in the definition of contrasts (see below) allowed assessment of linearly decreasing effects. Receipt of pain was modeled as distinct events. Epochs during which subjects actually received pain stimuli, ITIs and rating epochs were modeled as box car regressors. To retain degrees of freedom, the three runs were concatenated. Residual motion effects were corrected for by including the six estimated motion parameters for each subject as regressors in the model. Each regressor was convolved with the canonical hemodynamic response function. Calculation of voxelwise within-subject effects of linear combinations of the regressors yielded contrast images that were spatially smoothed (FWHM 10 $\mathrm{mm}$ ) and compared across subjects using one-sample $t$ tests. Interactions for linearly increasing (decreasing) responses are mathematically equivalent to interactions for linearly decreasing (increasing) responses.

Clusters with $>5$ voxels activated at a statistical threshold of $P=0.001$ are reported. A spherical region of interest (ROI) with a radius of $10 \mathrm{~mm}$ was defined in dorsal MPFC/ACC using coordinates $(-2 / 45 / 27)$ from Kalisch et al. (2005). In that study, mean MPFC/ACC coordinates from previous high resolution fMRI studies of anticipatory anxiety (Jensen et al., 2003; Ploghaus et al., 1999; Porro et al., 2002) had served to define a search volume for interactions between anticipatory anxiety and reappraisal, successfully identifying those. (Two further fMRI studies report MPFC/ ACC activation during pain anticipation but do not provide coordinates (Phelps et al., 2001; Wager et al., 2004)). The same coordinates have later been successfully used to identify anticipatory anxiety-related activation in another study from our laboratory (Kalisch et al., in press). Thus, the chosen coordinates represent a focus of anticipatory anxiety-related activity observed across a large number of studies, and the represented MPFC/ACC area can be regarded as central to anticipatory anxiety. Correction for multiple comparisons following Gaussian random field theory was limited to this region of interest.

To illustrate group effect sizes in selected voxels, mean parameter estimates from the main effect of a second-level oneway analysis of variance over the four experimental conditions were used. To test whether activation patterns in voxels showing a significant interaction were consistent with a reduction of anxietyrelated activity by cognitive load (i.e., whether they showed a significant simple main effect of Anxiety (Anxiety/Low Load > Noanxiety/Low Load) and a significant simple main effect of cognitive load under Anxiety (Anxiety/Low Load > Anxiety/High Load)), post hoc $t$ tests for the relevant contrasts were calculated within SPSS11 using single subject parameter estimates from the ANOVA (threshold $P=0.05$, one-tailed). Anatomical localization was carried out with reference to the atlas of Duvernoy (1999). Coordinates follow MNI conventions.

\section{Results}

Anticipation of pain increased subjective anxiety (contrast Anxiety $>$ No-anxiety, i.e., main effect of Anxiety: $F(1,13)=$ 228.8, $P<0.001$; Fig. 2a) and stress ratings $(F(1,12)=21.43, P=$ 0.001 ; Fig. 2b) and was associated with a trend-level increase in effort ratings $(F(1,13)=3.31, P=0.092$; Fig. 2c). Anticipation also caused a strong trend-level increase in tonic heart rate levels (HRL; $F(1,12)=4.2, P=0.062$; Fig. $2 \mathrm{~d})$ and in initial phasic heart rate responses (HRR; Fig. 2e) to the onset of pain cues (see Kalisch et al., 2005); $(1,12)=4.5, P=0.055)$. This is consistent with an anxiety response involving both the bodily and the subjective experiential level.

Increasing cognitive load prolonged reaction times (contrast High Load (2-back) > Low Load (0-back), i.e., main effect of Load: $F(1,13)=34.5, P<0.001$; Table 1). Due to the design of the 0 -back task (see Materials and methods), accuracy data could not be compared between the two difficulty levels. High cognitive load was not associated with higher subjective anxiety $(F(1,13)=0.14$, $P=0.72$; Fig. 2a). In contrast, subjective stress $(F(1,12)=17.15$, $P=0.001$; Fig. 2b) and effort ratings $(F(1,13)=26.02, P<0.001$; Fig. 2c) were increased as were both $\operatorname{HRL}(F(1,12)=6.1, P=$ 0.029; Fig. 2d) and HRR $(F(1,12)=4.9, P=0.047$; Fig. 2e). Thus, performing the 2-back task was more demanding and effortful than performing the 0 -back task, indicating that cognitive resources were required in the high load condition. Within a resource model of cognitive function, this implies that less resources were available for high-level appraisal, supporting our basic assumption.

There were no interactions between factors Anxiety and Load at the behavioral level. Anticipation of pain neither influenced n-back task reaction times (main effect of Anxiety: $F(1,13)=0.55, P=$ 0.47 ; interaction: $F(1,13)=0.21, P=0.65$; Table 1 ) nor accuracy (Table 2), showing that anticipatory anxiety did not interfere with 2back task performance. Thus, sufficient cognitive resources were allocated to the task even in the face of disturbing emotional stimulation. Likewise, no interactions were observed in subjective anxiety ( $P=0.223$; Fig. 2a), stress ( $P=0.703$; Fig. 2 b) and effort ratings $(P=0.748$; Fig. 2 c), HRL $(P=0.617$; Fig. 2 d) and HRR ( $P=0.889$; Fig. 2e). Anxiety causes increased reactivity to noxious stimuli (Epstein and Clarke, 1970; Kalisch et al., 2005; Ploghaus et al., 2001). Pain reactivity can thus be used as surrogate physiological indicator of anxiety. There was no significant difference in the phasic heart rate responses to pain stimuli as a function of load ( $P=0.263$; not shown). Furthermore, there were no significant reductions in neural responses to pain under high load (contrast pain Anxiety/Low Load $>$ pain $_{\text {Anxiety/High Load; see Materials }}$ and methods), in agreement with an absence of interactions between Anxiety and Load in subjective and autonomic measures. 


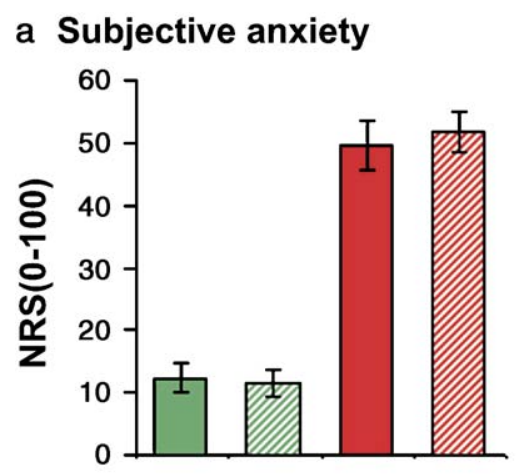

d HRL

b Subjective stress
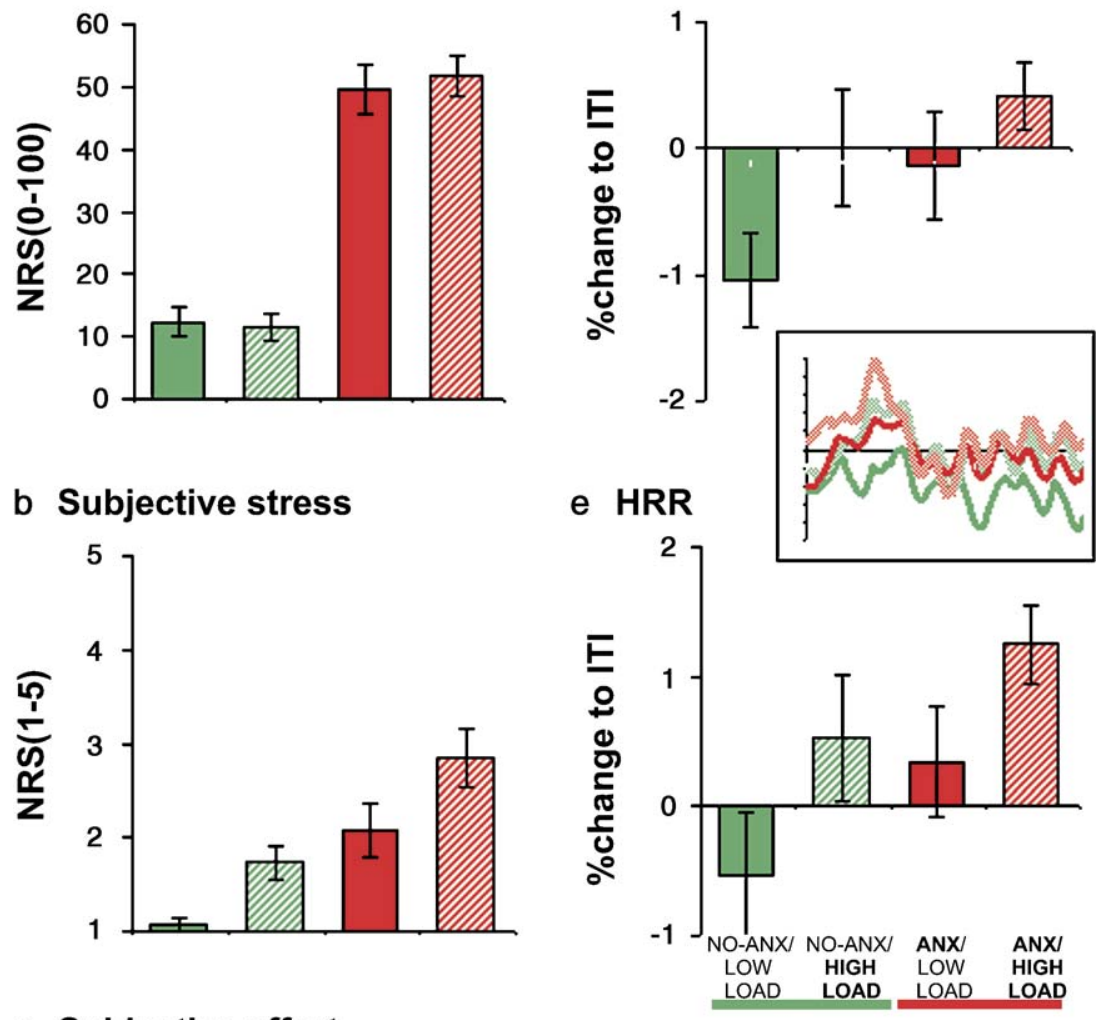

\section{c Subjective effort}

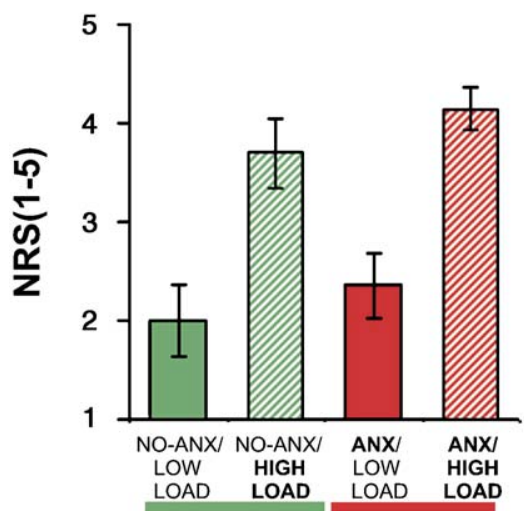

Fig. 2. Behavior. Interactions between Anxiety and Load were absent from subjective ratings of anxiety (a), stress (b) and effort (c) as well as from heart rate levels (HRL, d) and the phasic heart rate responses to onset of an anticipatory period (HRR, e).

The behavioral data thus show the predicted dissociation between high-level-appraisal-related activity (reduced under high load) and autonomic activity and subjective feelings (which were not affected by the load manipulation). This allows us to specifically relate any attenuation of anxiety-induced MPFC/

Table 1

Reaction times [ms]

\begin{tabular}{llll}
\hline $\begin{array}{l}\text { No-anxiety/ } \\
\text { Low Load }\end{array}$ & $\begin{array}{l}\text { No-anxiety/ } \\
\text { High Load }\end{array}$ & $\begin{array}{l}\text { Anxiety/ } \\
\text { Low Load }\end{array}$ & $\begin{array}{l}\text { Anxiety/ } \\
\text { High Load }\end{array}$ \\
\hline $423.29 \pm 27.03$ & $535.9 \pm 25.39$ & $428.5 \pm 19.82$ & $545.5 \pm 20.91$ \\
\hline
\end{tabular}

Reaction times were increased in the High Load condition. Anxiety did not influence reaction times. Values: mean \pm SEM.
ACC activation in the Anxiety/High Load relative to the Anxiety/Low Load condition to high-level appraisal, as predicted in our a priori hypothesis.

We therefore tested for an interaction of the type (Anxiety $>$ No-anxiety $)_{\text {Low Load }}>(\text { Anxiety }>\text { No-anxiety })_{\text {High Load }}$ in a predefined region of interest (see Materials and methods). Identified voxels had to fulfil the additional criteria of showing simple main effects of both Anxiety (Anxiety/Low Load $>$ Noanxiety/Low Load) and Load under Anxiety (Anxiety/High Load < Anxiety/Low Load) to provide evidence consistent with attenuation of anxiety-related activity. An activation peak meeting all criteria was found in MPFC/ACC with its peak located in the left paracingulate cortex (Brodmann area 32) for a tonic anxiety response (Fig. 3). No overall main effect of Anxiety was evident 
Table 2

Accuracy

\begin{tabular}{lccc}
\hline & No-anxiety/High Load & Anxiety/High Load & $P$ \\
\hline \% false positives & $0.52 \pm 0.21$ & $0.56 \pm 0.21$ & 0.90 \\
\% false negatives & $28.2 \pm 6.45$ & $21.56 \pm 4.26$ & 0.40 \\
\% true positives & $71.48 \pm 6.42$ & $77.36 \pm 4.25$ & 0.45 \\
\% true negatives & $99.15 \pm 0.35$ & $99.3 \pm 0.22$ & 0.73 \\
\% omissions & $0.32 \pm 0.26$ & $0.22 \pm 0.15$ & 0.73 \\
\hline
\end{tabular}

There were no differences in performance of the 2-back task (High Load condition) between the No-anxiety and Anxiety conditions. Values: mean \pm SEM. Paired $t$ test, 2-tailed.

within this region of interest. We therefore suggest that this dorsal MPFC/ACC area contributes to high-level appraisal.

Outside this region of interest, we also found effects in the same contrast within lateral prefrontal and parietal cortex $(P<0.001$, Fig. 4; Supplementary Table 1). The load manipulation thus affected an entire network of areas classically linked to attention and awareness (Nobre, 2001; Pessoa et al., 2003; Rees et al., 2002; Taylor et al., 2003). This further supports our conclusion that we successfully targeted a cognitive process dependent on attentional and working memory resources. No interactions of the type (High Load $>$ Low Load $)_{\text {No-anxiety }}>(\text { High Load }>\text { Low Load })_{\text {Anxiety }}$ were observed, paralleling the absence of effects of Anxiety on 2-back task performance. Main effects of Anxiety and Load are shown in Supplementary Figs. 1 and 2.

\section{Discussion}

We show that dorsal MPFC/ACC is implicated in high-level appraisal. Our results extend earlier findings (Johnson et al., 2002;
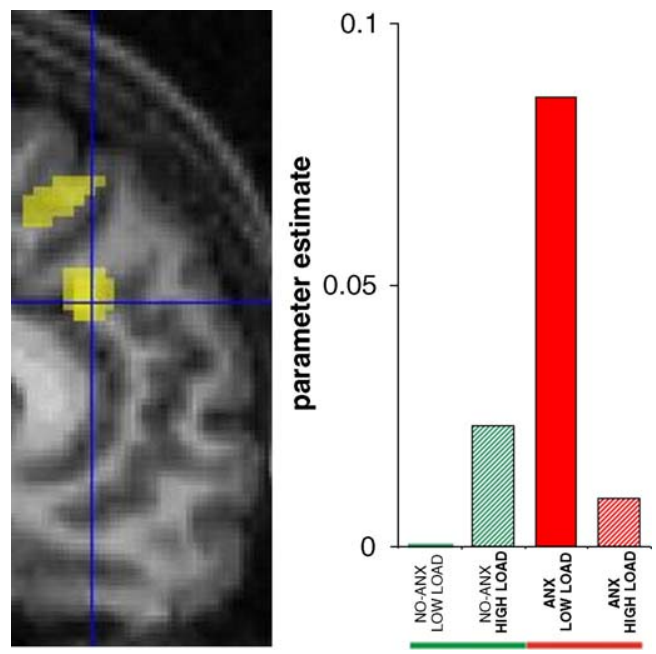

Fig. 3. Modulation of anxiety-related activity as a function of cognitive load indicates a high-level appraisal-specific function for dorsal MPFC/ ACC. A cluster within dorsal MPFC/ACC exhibited an interaction pattern consistent with reduction of anxiety-related activity during the 2-back task (High Load) relative to the 0 -back task (Low Load). Peak at $-8 / 38 / 28$ (haircross), $z=3.16, P=0.031$ small volume-corrected, tonic response. Display threshold $P=0.01$ uncorrected. Activation superimposed on one subject's normalized structural image. The more dorsal cluster was located outside the search volume and did not meet criteria fixed for reduction of anxiety-related activity (see Materials and methods). NO-ANX: Noanxiety, ANX: Anxiety.

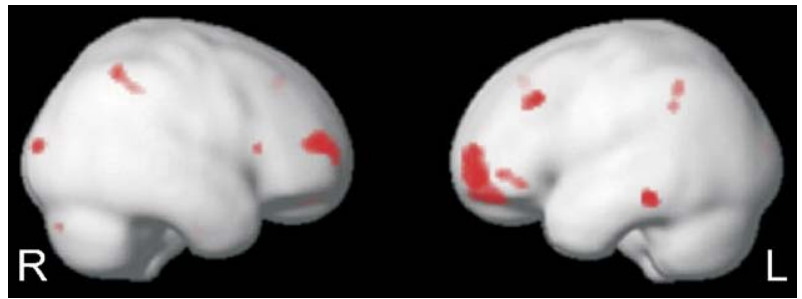

Fig. 4. A lateral cortical network implicated in high-level appraisal. Shown are tonic interactions of the type (Anxiety $>$ No-anxiety) Low Load $>$ (Anxiety $>$

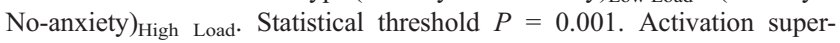
imposed on a canonical normalized structural image.

Lane et al., 1997, 1998; Simpson et al., 2001) and confirm a theoretical model of appraisal (Lewis, 2005). A crucial feature of our study is that we carefully controlled selective attentional deployment and controlled for confounds that may arise from correlated low-level appraisal processes or response generation activity. The findings are in agreement with observations of affective blunting following MPFC/ACC lesions (reviewed in Devinsky et al. (1995) and Cohen et al. (1999)). The left-sided focus of MPFC/ACC activity is most likely due to pain stimulus application to the right hand (Kalisch et al., 2005).

Anticipatory anxiety paradigms reproducibly activate the rostral medial prefrontal and anterior cingulate cortices (Jensen et al., 2003; Ploghaus et al., 1999; Porro et al., 2002). Rostral ACC activation is also observed when anxiety is induced by medication, visual imagery or recall of anxiety-provoking situations in normal subjects as well as by symptom provocation in anxiety patients (reviewed in Brody et al., 2001). Improvement in anxiety symptoms after pharmacological or psychotherapy is associated with decreases in rostral ACC resting blood flow (Brody et al., 2001). Finally, ACC volume and trait anxiety are positively correlated (Pujol et al., 2002). Although there is a considerable degree of variance in terms of the location of activation foci within the MPFC/ACC, these findings generally highlight the important role of the MPFC/ACC in anxiety. Other emotions like sadness (reviewed in Brody et al., 2001) or happiness (reviewed in Phan et al., 2002) also activate the MPFC/ACC. In fact, the MPFC/ACC is the brain region most consistently activated across different emotions (Phan et al., 2002), suggesting some shared function which may be provided by high-level appraisal. Interestingly, in a study by Liotti et al. (2000), no MPFC/ACC activation was found while subjects focused their awareness on previously induced feelings of anxiety or sadness when this condition was compared to a condition requiring focusing on feelings of relaxation, i.e., when high-level appraisal was engaged in the control condition as well.

A wider prefrontal-parietal network seems to complement an MPFC/ACC function in high-level appraisal. In particular, we observed an extended cluster in bilateral antero-lateral PFC, encompassing or close to regions previously implicated in reappraisal of and self distraction from anxiety (Kalisch et al., 2005, in press) and other emotional situations (Beauregard et al., 2001; Levesque et al., 2003, 2004; Ochsner et al., 2002, 2004; Phan et al., 2005; Schaefer et al., 2003).

Our data do not allow us to infer whether MPFC/ACC activity during high-level appraisal simply reflects conscious awareness of low-level appraisal outcomes and/or the emotion-eliciting stimuli or whether the MPFC/ACC actually performs high-level operations on these outcomes. We note, however, that MPFC/ACC activity is attenuated while antero-lateral PFC activity is enhanced during 
successful reappraisal of anticipatory anxiety (Kalisch et al., 2005). Furthermore, during that study, anxiety-related activity in MPFC/ $\mathrm{ACC}$ was phasic, i.e., consisting in a brief increase in activation at the onset of an anticipatory period, while reappraisal-related activity in right antero-lateral PFC increased linearly during anticipatory periods. It thus seems that MPFC/ACC activity can be regulated by lateral $\mathrm{PFC}$ and precedes lateral $\mathrm{PFC}$ activity during the course of an emotional reaction. (MPFC/ACC activity in the present study was tonic, presumably related to continuous presentation of anxiogenic stimuli (the red dots, Fig. 1), as opposed to phasic presentations in Kalisch et al. (2005), and to the absence of MPFC/ACC-attenuating reappraisal.) On the basis of these data, it is conceivable that the MPFC/ACC mediates attentional functions (Lewis, 2005), allowing low-level appraisal outcomes to gain access to consciousness. By contrast, the lateral PFC may use the conscious representations generated with the help of the MPFC/ACC for controlled appraisal.

Another possible distinction between MPFC/ACC and lateral PFC function in high-level appraisal is that the MPFC/ACC provides for schema-based appraisal, whereas the lateral PFC provides for conceptual-propositional appraisal (Leventhal and Scherer, 1987). Schemata contain thoughts, feelings, sensory-type representations and response programs that were part of similar emotional episodes in the past and guide appraisal and responding when activated by a matching stimulus (Lang, 1985; Foa and Kozak, 1986). Conceptual appraisal, by contrast, is based on propositionally organized memory structures and allows for reflexive, rather than automatic, evaluation (Leventhal and Scherer, 1987). Conceptual appraisal by its very nature depends on controlled processing. Whether schema-based appraisal, on the other hand, can also be conceptualized as a high-level appraisal is unclear. Schematic appraisal is often seen as implicit and automatic but also associated with "primal thinking" (Beck and Clark, 1997) or "mindless emoting" (Teasdale, 1999), activities that would appear to draw on working memory resources. In Scherer's account (Scherer, 2001), schematic appraisal, though automatic, operates on the contents of sensory stores, i.e., conscious representations. A possible role for the $\mathrm{MPFC} / \mathrm{ACC}$ in schematic appraisal is supported by a recent study reporting a dissociation between MPFC/ACC (albeit more ventral) and lateral PFC depending on whether subjects use aware schematic ("metaphoric", "holistic") or propositional-analytic appraisals (Schaefer et al., 2003), respectively. MPFC/ACC attenuation and lateral PFC enhancement during reappraisal of anxiety (Kalisch et al., 2005) could accordingly be interpreted as a switch from "mindless emoting" to some more elaborate appraisal.

A further possible implication of our model is that rostral ACC activity sometimes observed during situations of error and/or conflict (Dehaene et al., 2003; Ridderinkhof et al., 2004) is equal to a conscious negative appraisal process. Importantly, high-level appraisal of conflict or error (and thus ACC activity) may vary according to contextual factors such as familiarity, motivational relevance and mood. Indeed, there is strong evidence for such contextual modulatory influences on electrophysiological correlates of error/conflict (reviewed in Bush et al., 2000 and Lewis, 2005). On this basis and building on the work by Luu et al. (2003), Lewis (2005) has suggested that error-related potentials are generated by large-scale synchronized theta oscillations coupling the MPFC/ACC to other cortical and subcortical areas, allowing for attended evaluation of erroneous actions ("mini-appraisals").

Little has been known about the neurobiological basis of highlevel appraisal of emotional material in humans. In this study, we have clearly dissociated high-level appraisal from accompanying low-level appraisal and response generation processes using a psychological manipulation targeting controlled processing resources required for high-level appraisal while concurrently measuring low-level appraisal outcome. Our analysis allows us to ascribe to dorsal MPFC/ACC a mediating role in high-level appraisal. More generally, our data provide neurobiological evidence for a theoretical distinction between low-level and high-level appraisal.

\section{Acknowledgments}

We thank P. Allen, O. Josephs, P. Aston, E. Featherstone and B. Seymour for help with experiments and K. Friston for help with statistics. This work was supported by grants from the Marie Curie programme (RK), Deutsche Forschungsgemeinschaft (KW) and by Wellcome Trust Programme grants to HDC and RJD.

\section{Appendix A. Supplementary data}

Supplementary data associated with this article can be found in the online version at doi:10.1016/j.neuroimage.2005.11.011.

\section{References}

Ashburner, J., Friston, K., Penny, W., 2004. Imaging neuroscience-Theory and analysis. In: Frackowiak, R.S.J., Friston, K., Frith, C.D., Dolan, R.J., Price, C.J., Zeki, S., Ashburner, J., Penny, W. (Eds.), Human Brian Function, 2nd ed. Academic Press, San Diego, pp. 599-1104.

Beauregard, M., Levesque, J., Bourgouin, P., 2001. Neural correlates of conscious self-regulation of emotion. J. Neurosci. 21, RC165.

Beck, A.T., Clark, D.A., 1997. An information processing model of anxiety: automatic and strategic processes. Behav. Res. Ther. 35, 49-58.

Blackwood, N.J., Bentall, R.P., ffytche, D.H., Simmons, A., Murray, R.M., Howard, R.J., 2004. Persecutory delusions and the determination of self-relevance: an fMRI investigation. Psychol. Med. 34, 591-596.

Broadbent, D.E., Cooper, P.F., FitzGerald, P., Parkes, K.R., 1982. The Cognitive Failures Questionnaire (CFQ) and its correlates. Br. J. Clin. Psychol. 21, 1-16.

Brody, A.L., Saxena, S., Mandelkern, M.A., Fairbanks, L.A., Ho, M.L., Baxter, L.R., 2001. Brain metabolic changes associated with symptom factor improvement in major depressive disorder. Biol. Psychiatry 50, $171-178$.

Bush, G., Luu, P., Posner, M.I., 2000. Cognitive and emotional influences in anterior cingulate cortex. Trends Cogn. Sci. 4, 215-222.

Christianson, S.A., 1992. Emotional stress and eyewitness memory: a critical review. Psychol. Bull. 112 (2), 284-309.

Chua, P., Krams, M., Toni, I., Passingham, R., Dolan, R., 1999. A functional anatomy of anticipatory anxiety. NeuroImage 9, 563-571.

Clore, G.L., Parrott, W.G., 1991. Moods and their vicissitudes: thoughts and feelings as information. In: Forgas, J.P. (Ed.), Emotion and Social Judgment. Pergamon, Oxford, pp. 107-123.

Cohen, R.A., Kaplan, R.F., Zuffante, P., Moser, D.J., Jenkins, M.A., Salloway, S., Wilkinson, H., 1999. Alteration of intention and self-initiated action associated with bilateral anterior cingulotomy. J. Neuropsychiatry Clin. Neurosci. 11, 444-453.

Critchley, H.D., 2004. The human cortex responds to an interoceptive challenge. Proc. Natl. Acad. Sci. U. S. A. 101, 6333-6334.

Dayan, P., Abbott, L.F., 2001. Classical Conditioning and Reinforcement Learning. MIT Press, Cambridge, MA, pp. 331-358.

Dehaene, S., Artiges, E., Naccache, L., Martelli, C., Viard, A., Schurhoff, 
F., Recasens, C., Martinot, M.L., Leboyer, M., Martinot, J.L., 2003. Conscious and subliminal conflicts in normal subjects and patients with schizophrenia: the role of the anterior cingulate. Proc. Natl. Acad. Sci. U. S. A. 100, 13722-13727.

Deichmann, R., Gottfried, J.A., Hutton, C., Turner, R., 2003. Optimized EPI for fMRI studies of the orbitofrontal cortex. NeuroImage 19, $430-441$.

Deichmann, R., Schwarzbauer, C., Turner, R., 2004. Optimisation of the 3D MDEFT sequence for anatomical brain imaging: technical implications at 1.5 and $3 \mathrm{~T}$. NeuroImage $21,757-767$.

Devinsky, O., Morrell, M.J., Vogt, B.A., 1995. Contributions of anterior cingulate cortex to behaviour. Brain 118, 279-306.

Duvernoy, H.M., 1999. The Human Brain: Surface, Blood Supply, and Three-Dimensional Sectional Anatomy, 2nd ed. Springer, Wien.

Epstein, S., Clarke, S., 1970. Heart rate and skin conductance during experimentally induced anxiety: effects of anticipated intensity of noxious stimulation and experience. J. Exp. Psychol. 84, 105-112.

Epstein, S., Roupenian, A., 1970. Heart rate and skin conductance during experimentally induced anxiety: the effect of uncertainty about receiving a noxious stimulus. J. Pers. Soc. Psychol. 16, 20-28.

Erdelyi, M.H., Appelbaum, A.G., 1973. Cognitive masking: the disruptive effect of an emotional stimulus upon the perception of contiguous neutral material. Bull. Psychon. Soc. 1, 59-61.

Fichtenholtz, H.M., Dean, H.L., Dillon, D.G., Yamasaki, H., McCarthy, G., LaBar, K.S., 2004. Emotion-attention network interactions during a visual oddball task. Brain Res. Cogn. Brain Res. 20, 67-80.

Foa, E.B., Kozak, M.J., 1986. Emotional processing of fear: exposure to corrective information. Psychol. Bull. 99, 20-35.

Fossati, P., Hevenor, S.J., Graham, S.J., Grady, C., Keigthley, M.L., Craik, F., 2003. In search of the emotional self: an FMRI study using positive and negative emotional words. Am. J. Psychiatry 160, $1938-1945$

Frijda, N.H., 1993. The place of appraisal in emotion. Cogn. Emot. 7, $357-387$.

Gross, J.J., John, O.P., 2003. Individual differences in two emotion regulation processes: implications for affect, relationships, and wellbeing. J. Pers. Soc. Psychol. 85, 348-362.

Hare, R.D., 1966. Denial of threat and emotional response to impending painful stimulation. J. Consult. Psychol. 30, 359-361.

Houston, B.K., Holmes, D.S., 1974. Effect of avoidant thinking and reappraisal for coping with threat involving temporal uncertainty. J. Pers. Soc. Psychol. 30, 382-388.

Jensen, J., McIntosh, A.R., Crawley, A.P., Mikulis, D.J., Remington, G., Kapur, S., 2003. Direct activation of the ventral striatum in anticipation of aversive stimuli. Neuron 40, 1251-1257.

Johnson, S.C., Baxter, L., Wilder, L., Heiserman, J., Pipe, J., Prigatano, G., 2002. Neural correlates of self reflection. Brain 125, 1808-1814.

Kalisch, R., Wiech, K., Critchley, H.D., Seymour, B., O’Doherty, J.P., Oakley, D.A., Allen, P., Dolan, R.J., 2005. Anxiety reduction through detachment: subjective, physiological and neural effects. J. Cogn. Neurosci. 17, 874-883.

Kalisch, R., Wiech, K., Herrmann, K., Dolan, R.J., in press. Neural correlates of self distraction from anxiety and a process model of cognitive emotion regulation. J. Cogn. Neurosci.

Kelley, W.M., Macrae, C.N., Wyland, C.L., Caglar, S., Inati, S., Heatherton, T.F., 2002. Finding the self? An event-related fMRI study. J. Cogn. Neurosci. 14, 785-794.

Kunst-Wilson, W.R., Zajonc, R.B., 1980. Affective discrimination of stimuli that cannot be recognized. Science 207, 557-558.

Lane, R.D., Fink, G.R., Chau, P.M., Dolan, R.J., 1997. Neural activation during selective attention to subjective emotional responses. NeuroReport 8, 3969-3972

Lane, R.D., Reiman, E.M., Axelrod, B., Yun, L.S., Holmes, A., Schwartz, G.E., 1998. Neural correlates of levels of emotional awareness. Evidence of an interaction between emotion and attention in the anterior cingulate cortex. J. Cogn. Neurosci. 10, 525-535.

Lang, P.J., 1985. The cognitive psychophysiology of emotion: fear and anxiety. In: Tuma, A.H., Maser, J.D. (Eds.), Anxiety and the Anxiety Disorders. Erlbaum, Hillsdale, NJ, pp. 131-170.

Lazarus, R.S., 1966. Psychological Stress and the Coping Process. McGraw-Hill.

Lazarus, R.S., McCleary, R.A., 1951. Autonomic discrimination without awareness: a study of subception. Psychol. Rev. 58, 113-122.

LeDoux, J.E., 1996. The Emotional Brain. Touchstone, New York, NY.

Leventhal, H., Scherer, K.R., 1987. The relationship of emotion to cognition: a functional approach to a semantic controversy. Cogn. Emot. 1, 3-28.

Levesque, J., Eugene, F., Joanette, Y., Paquette, V., Mensour, B., Beaudoin, G., Leroux, J.M., Bourgouin, P., Beauregard, M., 2003. Neural circuitry underlying voluntary suppression of sadness. Biol. Psychiatry $53,502-510$

Levesque, J., Joanette, Y., Mensour, B., Beaudoin, G., Leroux, J.M., Bourgouin, P., Beauregard, M., 2004. Neural basis of emotional selfregulation in childhood. Neuroscience 129, 361-369.

Lewis, M.D., 2005. Bridging emotion theory and neurobiology through dynamic systems modeling. Behav. Brain Sci. 28, 169-194.

Liotti, M., Mayberg, H.S., Brannan, S.K., McGinnis, S., Jerabek, P., Fox, P.T., 2000. Differential limbic-cortical correlates of sadness and anxiety in healthy subjects: implications for affective disorders. Biol. Psychiatry 48, 30-42.

Luu, P., Tucker, D.M., Derryberry, D., Reed, M., Poulsen, C., 2003. Electrophysiological responses to errors and feedback in the process of action regulation. Psychol. Sci. 14, 47-53.

Monat, A., Averill, J.R., Lazarus, R.S., 1972. Anticipatory stress and coping reactions under various conditions of uncertainty. J. Pers. Soc. Psychol. $24,237-253$

Moray, N., 1959. Attention in dichotic listening: affective cues and the influence of instruction. Quart. J. Exp. Psychol. 11, 56-60.

Nielsen, S.L., Sarason, I.G., 1981. Emotion, personality and selective attention. J. Pers. Soc. Psychol. 41, 945-960.

Nobre, A.C., 2001. The attentive homunculus: now you see it, now you don't. Neurosci. Biobehav. Rev. 25, 477-496.

Ochsner, K.N., Bunge, S.A., Gross, J.J., Gabrieli, J.D., 2002. Rethinking feelings: an fMRI study of the cognitive regulation of emotion. J. Cogn. Neurosci. 14, 1215-1229.

Ochsner, K.N., Ray, R.D., Cooper, J.C., Robertson, E.R., Chopra, S., Gabrieli, J.D., Gross, J.J., 2004. For better or for worse: neural systems supporting the cognitive down- and up-regulation of negative emotion. NeuroImage 23, 483-499.

Öhman, A., 1993. Fear and anxiety as emotional phenomena: clinical phenomenology, evolutionary perspectives, and information-processing mechanisms. In: Lewis, M., Haviland, J.M. (Eds.), Handbook of Emotions, pp. 511-536.

Öhman, A., 2005. The role of the amygdala in human fear: automatic detection of threat. Psychoneuroendocrinology 30, 953-958.

Pessoa, L., Kastner, S., Ungerleider, L.G., 2003. Neuroimaging studies of attention: from modulation of sensory processing to top-down control. J. Neurosci. 23, 3990-3998.

Phan, K.L., Wager, T., Taylor, S.F., Liberzon, I., 2002. Functional neuroanatomy of emotion: a meta-analysis of emotion activation studies in PET and fMRI. NeuroImage 16, 331-348.

Phan, K.L., Taylor, S.F., Welsh, R.C., Ho, S.H., Britton, J.C., Liberzon, I., 2004. Neural correlates of individual ratings of emotional salience: a trial-related fMRI study. NeuroImage 21, 768-780.

Phan, K.L., Fitzgerald, D.A., Nathan, P.J., Moore, G.J., Uhde, T.W., Tancer, M.E., 2005. Neural substrates for voluntary suppression of negative affect: a functional magnetic resonance imaging study. Biol. Psychiatry $57,210-219$

Phelps, E.A., O'Connor, K.J., Gatenby, J.C., Gore, J.C., Grillon, C., Davis, M., 2001. Activation of the left amygdala to a cognitive representation of fear. Nat. Neurosci. 4, 437-441.

Ploghaus, A., Tracey, I., Gati, J.S., Clare, S., Menon, R.S., Matthews, P.M., Rawlins, J.N., 1999. Dissociating pain from its anticipation in the human brain. Science 284, 1979-1981. 
Ploghaus, A., Narain, C., Beckmann, C.F., Clare, S., Bantick, S., Wise, R., Matthews, P.M., Rawlins, J.N., Tracey, I., 2001. Exacerbation of pain by anxiety is associated with activity in a hippocampal network. J. Neurosci. 21, 9896-9903.

Porro, C.A., Baraldi, P., Pagnoni, G., Serafini, M., Facchin, P., Maieron, M., Nichelli, P., 2002. Does anticipation of pain affect cortical nociceptive systems? J. Neurosci. 22, 3206-3214.

Pujol, J., Lopez, A., Deus, J., Cardoner, N., Vallejo, J., Capdevila, A., Paus, T., 2002. Anatomical variability of the anterior cingulate gyrus and basic dimensions of human personality. NeuroImage 15, 847-855.

Rees, G., Kreiman, G., Koch, C., 2002. Neural correlates of consciousness in humans. Nat. Rev., Neurosci. 3, 261-270.

Reisenzein, R., 2001. Appraisal processes conceptualized from a schematheoretic perspective. In: Scherer, K.R., Schorr, A., Johnstone, T. (Eds.), Appraisal Processes in Emotion: Theory, Methods, Research, pp. 187-201.

Ridderinkhof, K.R., Ullsperger, M., Crone, E.A., Nieuwenhuis, S., 2004. The role of the medial frontal cortex in cognitive control. Science 306, $443-447$

Robinson, M.D., 1998. Running from William James' bear: a review of preattentive mechanisms and their contributions to emotional experience. Cogn. Emot. 12, 667-696.

Robles, R., Smith, R., Carver, C.S., Wellens, A.R., 1987. Influence of subliminal visual images on the experience of anxiety. Pers. Soc. Psychol. Bull. 13, 399-410.

Sander, D., Grandjean, D., Scherer, K.R., 2005. A systems approach to appraisal mechanisms in emotion. Neural Netw. 18, 317-352.

Schaefer, A., Collette, F., Philippot, P., van der, L.M., Laureys, S., Delfiore, G., Degueldre, C., Maquet, P., Luxen, A., Salmon, E., 2003. Neural correlates of "hot" and "cold" emotional processing: a multilevel approach to the functional anatomy of emotion. NeuroImage $18,938-949$.

Scherer, K.R., 1984. On the nature and function of emotion: a component process approach. In: Scherer, K.R., Ekman, P. (Eds.), Approaches to Emotion, pp. 293-318.
Scherer, K.R., 2001. Appraisal considered as a process of multilevel sequential checking. In: Scherer, K.R., Schorr, A., Johnstone, T. (Eds.), Appraisal Processes in Emotion: Theory, Methods, Research, pp. $92-120$.

Schneider, W., Dumais, S.T., Shiffrin, R.M., 1984. Automatic and controlled processing and attention. In: Parasuraman, R., Davies, D.R. (Eds.), Varieties of Attention, pp. 1-27.

Seymour, B., O’Doherty, J.P., Dayan, P., Koltzenburg, M., Jones, A.K., Dolan, R.J., Friston, K.J., Frackowiak, R.S., 2004. Temporal difference models describe higher-order learning in humans. Nature 429, $664-667$.

Simpson Jr., J.R., Snyder, A.Z., Gusnard, D.A., Raichle, M.E., 2001. Emotion-induced changes in human medial prefrontal cortex: I. During cognitive task performance. Proc. Natl. Acad. Sci. U. S. A. 98, 683-687.

Smith, C.A., Kirby, L.D., 2001. Toward delivering on the promise of appraisal theory. In: Scherer, K.R., Schorr, A., Johnstone, T. (Eds.), Appraisal Processes in Emotion: Theory, Methods, Research, pp. 187-201.

Spielberger, C.D., 1983. State-Trait Anxiety Inventory (Form Y). Mind Garden, Redwood City.

Taylor, S.F., Phan, K.L., Decker, L.R., Liberzon, I., 2003. Subjective rating of emotionally salient stimuli modulates neural activity. NeuroImage $18,650-659$.

Teasdale, J.D., 1999. Emotional processing, three modes of mind and the prevention of relapse in depression. Behav. Res. Ther. 37 (Suppl. 1), S53-S77.

Vuilleumier, P., Armony, J.L., Clarke, K., Husain, M., Driver, J., Dolan, R.J., 2002. Neural response to emotional faces with and without awareness: event-related fMRI in a parietal patient with visual extinction and spatial neglect. Neuropsychologia 40, 2156-2166.

Wager, T.D., Rilling, J.K., Smith, E.E., Sokolik, A., Casey, K.L., Davidson, R.J., Kosslyn, S.M., Rose, R.M., Cohen, J.D., 2004. Placebo-induced changes in FMRI in the anticipation and experience of pain. Science $303,1162-1167$. 